This item was submitted to Loughborough's Research Repository by the author.

Items in Figshare are protected by copyright, with all rights reserved, unless otherwise indicated.

\title{
Structural model updating and prediction variability using Pareto optimal models
}

PLEASE CITE THE PUBLISHED VERSION

http://dx.doi.org/10.1016/j.cma.2008.04.010

PUBLISHER

(c) Elsevier

VERSION

AM (Accepted Manuscript)

LICENCE

CC BY-NC-ND 4.0

\section{REPOSITORY RECORD}

Christodoulou, Konstantinos, Evangelos Ntotsios, Costas Papadimitriou, and Panagiotis Panetsos. 2019. "Structural Model Updating and Prediction Variability Using Pareto Optimal Models". figshare. https://hdl.handle.net/2134/10761. 
This item was submitted to Loughborough's Institutional Repository (https://dspace.lboro.ac.uk/) by the author and is made available under the following Creative Commons Licence conditions.

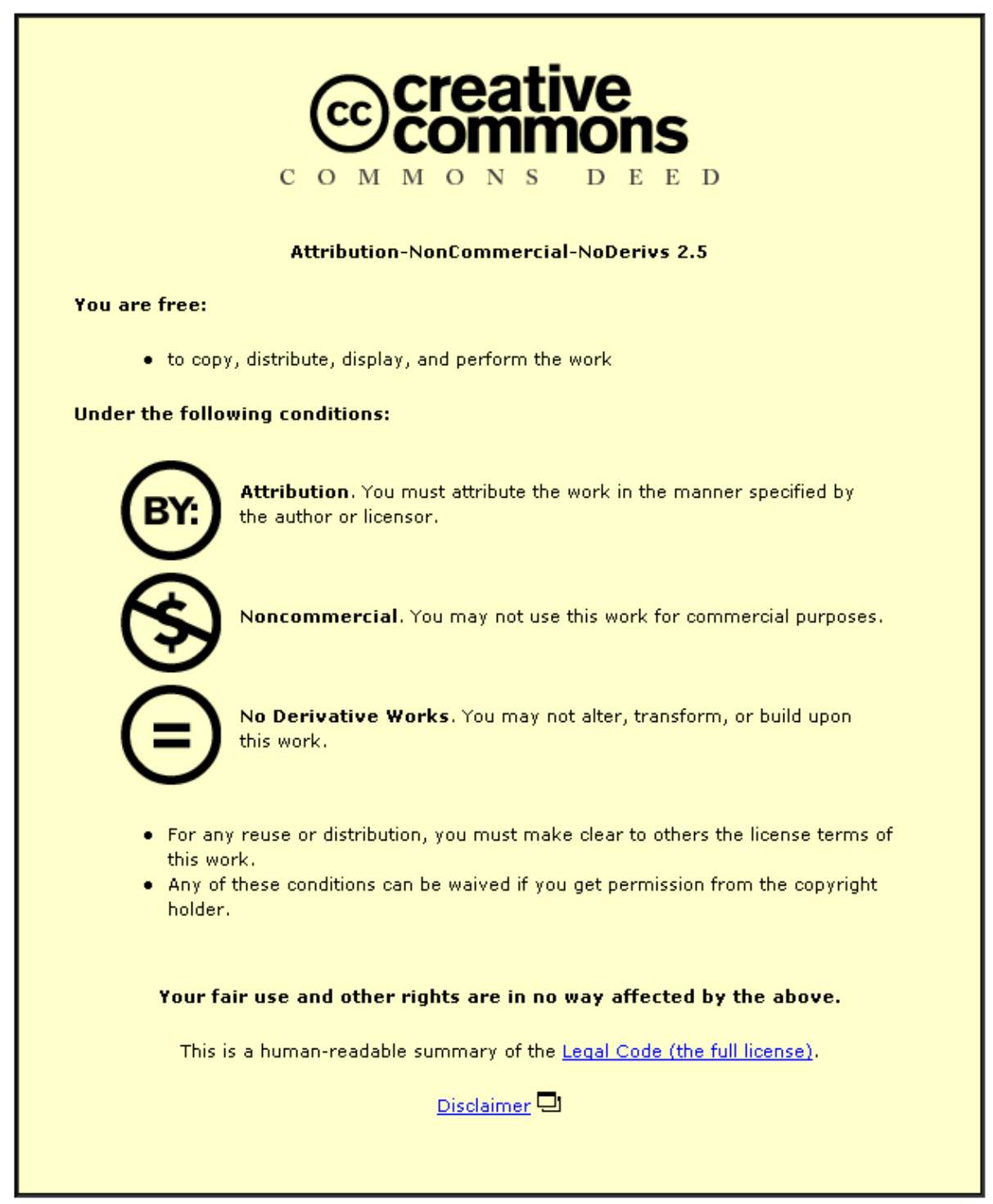

For the full text of this licence, please go to: http://creativecommons.org/licenses/by-nc-nd/2.5/ 


\title{
Structural model updating and prediction variability using Pareto optimal models
}

\author{
Konstantinos Christodoulou, Evaggelos Ntotsios, Costas Papadimitriou *, \\ Dept. of Mechanical and Industrial Engineering, University of Thessaly, Volos 38334, Greece \\ Panagiotis Panetsos \\ Egnatia Odos A.E., Capital Maintenance Department, GR 57001 Thermi, Greece
}

\begin{abstract}
A multi-objective identification method for structural model updating based on modal residuals is presented. The method results in multiple Pareto optimal structural models that are consistent with the experimentally measured modal data and the modal residuals used to measure the discrepancies between the measured and model predicted modal characteristics. These Pareto optimal models are due to uncertainties arising from model and measurement errors. The relation between the multi-objective identification method and the conventional single-objective weighted modal residuals method for model updating is investigated. Using this relation, an optimally weighted modal residuals method is also proposed to rationally select the most preferred model among the alternative multiple Pareto optimal models for further use in structural model prediction studies. Computational issues related to the reliable solution of the resulting multiobjective and single optimization problems are addressed. The model updating methods are compared and their effectiveness is demonstrated using experimental results obtained from a three-story laboratory structure tested at a reference and a mass modified configuration. The variability of the Pareto optimal models and their associated response prediction variability are explored using two structural model classes, a simple 3-DOF model class and a higher fidelity 546-DOF finite element model class. It is demonstrated that the Pareto optimal structural models and the corresponding response and reliability predictions may vary considerably, depending on the fidelity of the model class and the size of measurement errors.
\end{abstract}

Key Words: Model updating; Structural identification; Multi-objective optimization; Pareto optimality; Reliability

* Corresponding Author. Tel: (+30) 2421074006, Fax: (+30) 2421074012

Email address: costasp@uth.gr (Costas Papadimitriou) 


\section{Introduction}

Structural model updating methods (e.g. [1-6]) have been proposed in the past to reconcile mathematical models, usually discretized finite element models, with modal data obtained from experimental modal analysis. Each model updating method has its own advantages and shortcomings, but there is no universally acceptable methodology for treating the model updating problem. Comprehensive reviews of structural model updating methods can be found in the work by Mottershead and Friswell [2] and Doebling et al. [7]. The estimate of the optimal model is sensitive to uncertainties that are due to limitations of the mathematical models used to represent the behavior of the real structure, the presence of measurement noise from ambient excitations (e.g. wind, facilities and motion occupants in buildings [8]), and the processing errors in estimating the modal data. Also, optimal model estimates are sensitive to the number and type of measured modal data used in the reconciling process, as well as the norms used to measure the fit between measured and model predicted modal properties. The optimal structural models resulting from such methods can be used for improving the model response and reliability predictions, as well as accounting for the uncertainties in these predictions [9-11]. Moreover, these optimal structural models can be used for structural health monitoring applications [12-19] and structural control [20].

Structural model parameter estimation problems based on measured modal data (e.g $[2,21-23])$ are often formulated as weighted least-squares problems in which modal metrics, measuring the residuals between measured and model predicted modal properties, are build up into a single weighted modal residuals metric formed as a weighted average of the individual modal metrics using weighting factors. Standard optimization techniques are then used to find the optimal values of the structural parameters that minimize the single weighted residuals metric representing an overall 
measure of fit between measured and model predicted modal properties. Due to model error and measurement noise, the results of the optimization are affected by the values assumed for the weighting factors. The choice of the weighting factors depends on the model adequacy and the uncertainty in the available measured data, which are not known apriori. Different values of the weights result in different optimal models and consequently different predictions from the optimal models.

The model updating problem has recently been formulated in a multi-objective context [24] that allows the simultaneous minimization of the multiple modal metrics, eliminating the need for using arbitrary weighting factors for weighting the relative importance of each modal metric in the overall measure of fit. In contrast to the conventional weighted least-squares fit between measured and model predicted modal data, the multi-objective parameter estimation methodology provides multiple Pareto optimal structural models consistent with the data in the sense that the fit each Pareto optimal model provides in a group of measured modal properties cannot be improved without deteriorating the fit in at least one other modal group.

In this work, the multi-objective identification method based on modal data is revisited. In Section 2, the structural model updating problem using modal residuals is first formulated as a multi-objective optimization problem and then as a single-objective optimization with the single objective formed as a weighted average of the multiple objectives using weighting factors. Theoretical and computational issues arising in multiobjective identification are addressed and the correspondence between the multiobjective identification and the weighted modal residuals identification is established. Using this relation, the problem of rationally estimating the optimal values of the weights on which to base the model updating or, equivalently, selecting the most preferred structural model among the Pareto optimal models utilizing the available measured data 
is addressed in Section 3. A computationally efficient algorithm for simultaneously estimating these optimal weight values and the corresponding values of the parameters of the most preferred structural model is proposed. In addition, in Section 4, computational issues associated with solving the resulting multi-objective and single-objective optimization problems are addressed, including issues related to estimation of global optima, convergence of the proposed algorithms, and identifiability.

Finally, in Section 5, theoretical and computational issues are illustrated by applying the methodology for updating two model classes, a simple three degrees-of-freedom (DOF) model and a much higher fidelity finite element model class, using experimentally obtained modal data from a small-scaled three-story laboratory steel building structure tested at a reference and a mass modified configuration. Validation studies are performed to show the applicability of the methodologies, the advantages of the multi-objective identification, and the performance of the most preferred Pareto optimal model. Emphasis is given in investigating the variability of the Pareto optimal models and the variability of the response predictions from these Pareto optimal models. Comparisons between the results from a simple 3-DOF model class and a much higher fidelity finite element model class, are used to assess the effect of model error uncertainty on model updating and model response prediction variability.

\section{Model updating based on modal residuals}

\subsection{Preliminaries}

Let $D=\left\{\hat{\omega}_{r}^{(k)}, \hat{\phi}_{r}^{(k)} \in R^{N_{0}}, r=1, \cdots, m, k=1, \cdots, N_{D}\right\}$ be the measured modal data from a structure, consisting of modal frequencies $\hat{\omega}_{r}^{(k)}$ and modeshape components $\underline{\phi}_{r}^{(k)}$ at $N_{0}$ measured DOFs, where $m$ is the number of observed modes and $N_{D}$ is the number of modal data sets available. Consider a parameterized class of linear structural 
models used to model the dynamic behavior of the structure and let $\underline{\theta} \in R^{N_{\theta}}$ be the set of free structural model parameters to be identified using the measured modal data. Let also $\left\{\omega_{r}(\underline{\theta}), \underline{\phi}_{r}(\underline{\theta}) \in R^{N_{0}}, r=1, \cdots, m\right\}$ be the predictions of the modal frequencies and modeshape components at $N_{0}$ measured DOFs obtained for a particular value of the parameter set $\underline{\theta}$ by solving the eigenvalue problem corresponding to the model mass and stiffness matrices $M(\underline{\theta})$ and $K(\underline{\theta})$, respectively. The global matrices $M(\underline{\theta})$ and $K(\underline{\theta})$ depend on the values of the set $\underline{\theta}$ and are assembled from the element (or substructure) mass and stiffness matrices using a finite element analysis. Moreover, the measured DOFs $N_{0}$ are in most cases less than the model DOFs $N_{d}\left(N_{0} \leq N_{d}\right)$.

The objective in a modal-based structural identification methodology is to estimate the values of the parameter set $\underline{\theta}$ so that the modal data $\left\{\omega_{r}(\underline{\theta}), \underline{\phi}_{r}(\underline{\theta}), r=1, \cdots, m\right\}$ predicted by the linear class of models best matches, in some sense, the experimentally obtained modal data in $D$. For this, let

$$
J_{\omega_{r}}(\underline{\theta})=\frac{1}{N_{D}} \sum_{k=1}^{N_{D}} \frac{\left[\omega_{r}(\underline{\theta})-\hat{\omega}_{r}^{(k)}\right]^{2}}{\left[\hat{\omega}_{r}^{(k)}\right]^{2}} \quad \text { and } \quad J_{\underline{\phi}_{r}}(\underline{\theta})=\frac{1}{N_{D}} \sum_{k=1}^{N_{D}} \frac{\left\|\beta_{r}^{(k)} \underline{\phi}_{r}(\underline{\theta})-\underline{\hat{\phi}}_{r}^{(k)}\right\|^{2}}{\left\|\hat{\phi}_{r}^{(k)}\right\|_{N_{0}}^{2}}
$$

$r=1, \cdots, m$, be the measures of fit or residuals between the $N_{D}$ measured set of modal data and the model predicted modal data for the $r$-th modal frequency and modeshape components, respectively, where $\|\underline{z}\|^{2}=\underline{z}^{\mathrm{T}} \underline{z}$ is the usual Euclidian norm and $\|\underline{z}\|_{N_{0}}^{2}=\|\underline{z}\|^{2} / N_{0}$. Specifically, the objective $J_{\omega_{r}}(\underline{\theta})$ gives the mean of the fractional errors between the $r$-th measured modal frequencies and the $r$-th modal frequency predicted by the model class for a particular value of the parameter set $\underline{\theta}$. Similarly, the objective $J_{\underline{\phi}_{r}}(\underline{\theta})$ gives the mean of the normalized errors between the $r$-th measured 
modeshapes and the $r$-th modeshape predicted by the model class for a particular value of the parameter set $\underline{\theta}$. The scaling factor $\beta_{r}^{(k)} \equiv \beta_{r}^{(k)}(\underline{\theta})=\underline{\hat{\phi}}_{r}^{(k) T} \underline{\phi}_{r}(\underline{\theta}) /\left\|\underline{\phi}_{r}(\underline{\theta})\right\|^{2}$ is selected such that the measured modeshape $\hat{\phi}_{r}^{(k)}$ is closest to the model predicted modeshape $\beta_{r}^{(k)} \underline{\phi}_{r}(\underline{\theta})$ for a given value of $\underline{\theta}$, in the sense that the distance $\left\|\beta_{r}^{(k)} \underline{\phi}_{r}(\underline{\theta})-\underline{\phi}_{r}^{(k)}\right\|$ between the measured and the model predicted modeshapes is minimum. These errors are due to modeling error and measurement noise (e.g. $[8,9])$.

The measured modal properties are grouped into $n$ groups $g_{i}, i=1, \cdots, n$. Each group contains one or more modal properties. For the $i$ th group $g_{i}$, a norm $J_{i}(\underline{\theta})$ is introduced to measure the residuals of the difference between the measured values of the modal properties involved in the group and the corresponding modal values predicted from the model class for a particular value of the parameter set $\underline{\theta}$. The measure of fit in a modal group is taken as the sum of the individual measures of fit in (1) for the corresponding modal properties involved in the modal group. The grouping of the modal properties $\left\{\omega_{r}(\underline{\theta}), \underline{\phi}_{r}(\underline{\theta}), r=1, \cdots, m\right\}$ into $n$ groups and the selection of the measures of fit $J_{1}(\underline{\theta}), \cdots, J_{n}(\underline{\theta})$ are usually based on user preference. The modal properties assigned to each group are selected according to their type and the purpose of the analysis.

For demonstration purposes, a grouping scheme is next defined by grouping the modal properties into $n=2$ groups as follows. The first group contains all modal frequencies with the measure of fit $J_{1}(\underline{\theta})$ selected to represent the difference between the measured and the model predicted modal frequencies for all measured modes, while the second group contains the modeshape components for all measured modes with the measure of fit $J_{2}(\underline{\theta})$ selected to represents the difference between the measured and the 
model predicted modeshape components for all modes. Specifically, the $n=2$ measures of fit are given by

$$
J_{1}(\underline{\theta})=\sum_{r=1}^{m} J_{\omega_{r}}(\underline{\theta}) \quad \text { and } \quad J_{2}(\underline{\theta})=\sum_{r=1}^{m} J_{\underline{\phi}_{r}}(\underline{\theta})
$$

The aforementioned grouping scheme is used in the application section for demonstrating the features of the proposed model updating methodologies.

\subsection{Multi-objective identification}

The problem of model updating for identifying the model parameter values that give the best fit in all groups of modal properties can be formulated as a multi-objective optimization problem stated as follows [24]. Find the values of the structural parameter set $\underline{\theta}$ that simultaneously minimizes the objectives

$$
\underline{y}=\underline{J}(\underline{\theta})=\left(J_{1}(\underline{\theta}), \cdots, J_{n}(\underline{\theta})\right)
$$

where $\underline{\theta}=\left(\theta_{1}, \cdots, \theta_{N_{\theta}}\right) \in \Theta$ is the parameter vector, $\Theta$ is the parameter space, $\underline{y}=\left(y_{1}, \cdots, y_{n}\right) \in Y$ is the objective vector, and $Y$ is the objective space. For conflicting objectives $J_{1}(\underline{\theta}), \cdots, J_{n}(\underline{\theta})$, there is no single optimal solution, but rather a set of alternative solutions, known as Pareto optimal solutions, that are optimal in the sense that no other solutions in the parameter space are superior to them when all objectives are considered.

Using multi-objective terminology, the Pareto optimal solutions are the nondominating vectors in the parameter space $\Theta$, defined mathematically as follows. A vector $\underline{\theta} \in \Theta$ is said to be non-dominated regarding the set $\Theta$ if and only if there is no 
vector in $\Theta$ which dominates $\underline{\theta}$. A vector $\underline{\theta}$ is said to dominate a vector $\underline{\theta}^{\prime}$ if and only if

$$
J_{i}(\underline{\theta}) \leq J_{i}\left(\underline{\theta}^{\prime}\right) \quad \forall i \in\{1, \cdots, n\} \text { and } \exists j \in\{1, \cdots, n\} \text { such that } J_{j}(\underline{\theta})<J_{j}\left(\underline{\theta}^{\prime}\right)
$$

The set of objective vectors $\underline{y}=\underline{J}(\underline{\theta})$ corresponding to the set of Pareto optimal solutions $\underline{\theta}$ is called Pareto optimal front. The characteristics of the Pareto solutions are that the modal residuals cannot be improved in any modal group without deteriorating the modal residuals in at least one other modal group.

The multiple Pareto optimal solutions are due to modelling and measurement errors. The level of modelling and measurement errors affect the size and the distance from the origin of the Pareto front in the objective space, as well as the variability of the Pareto optimal solutions in the parameter space. The variability of the Pareto optimal solutions also depends on the overall sensitivity of the objective functions or, equivalently, the sensitivity of the modal properties, to model parameter values $\underline{\theta}$. Such variabilities were demonstrated for the case of two-dimensional objective space and one-dimensional parameter space in the work by Christodoulou and Papadimitriou [25]. In the application section, an example structure is used to demonstrate the effect of model error on the size and variability of the Pareto front and Pareto optimal solutions.

It should be noted that in the absence of modelling and measurement errors, there is an optimal value $\underline{\hat{\theta}}$ of the parameter set $\underline{\theta}$ for which the model based modal frequencies and modeshape components match exactly the corresponding measured modal properties. In this case, all objective functions $J_{1}(\underline{\hat{\theta}}), \cdots, J_{n}(\underline{\hat{\theta}})$ take the value of zero and, consequently, the Pareto front consists of a single point at the origin of the objective space. In particular, for identifiable problems [26-27], the solutions in the parameter space consist of one or more isolated points for the case of a single or multiple global 
optima, respectively. For non-identifiable problems [28-29], the Pareto optimal solutions form a lower dimensional manifold in the parameter space.

\subsection{Weighted modal residuals identification}

The parameter estimation problem is traditionally solved by minimizing the single objective

$$
J(\underline{\theta} ; \underline{w})=\sum_{i=1}^{n} w_{i} J_{i}(\underline{\theta})
$$

formed from the multiple objectives $J_{i}(\underline{\theta})$ using the weighting factors $w_{i} \geq 0$, $i=1, \cdots, n$, with $\sum_{i=1}^{n} w_{i}=1$. The objective function $J(\underline{\theta} ; \underline{w})$ represents an overall measure of fit between the measured and the model predicted modal characteristics. The relative importance of the modal residual errors in the selection of the optimal model is reflected in the choice of the weights. The results of the identification depend on the weight values used. The weight values depend on the adequacy of the model class used to represent structural behavior and the accuracy with which the measured modal data are obtained. However, the choice of weight values is arbitrary since the modeling error and the uncertainty in the measured data are usually not known apriori. Conventional weighted least squares methods assume equal weight values, $w_{1}=\cdots=w_{n}=1 / n$. This conventional method is referred herein as the equally weighted modal residuals method.

\subsection{Comparison between multi-objective and weighted modal residuals identification}

Formulating the parameter identification problem as a multi-objective minimization problem, the need for using arbitrary weighting factors for weighting the relative importance of the residuals $J_{i}(\underline{\theta})$ of a modal group to an overall weighted residuals 
metric is eliminated. An advantage of the multi-objective identification methodology is that all admissible solutions in the parameter space are obtained.

It can be readily shown that the optimal solution to the problem (5) is one of the Pareto optimal solutions. For this, let $\underline{\hat{\theta}}$ be the global optimal solution that minimizes the objective function $J(\underline{\theta} ; \underline{w})$ in (5) for given $\underline{w}$. Then this solution is also a Pareto optimal solution since otherwise there would exist another solution, say $\underline{\hat{\theta}}^{\prime}$, for which equation (4) will be satisfied for $\underline{\theta}=\underline{\hat{\theta}}^{\prime}$ and $\underline{\theta}^{\prime}=\underline{\hat{\theta}}$, that is, $J_{i}\left(\underline{\hat{\theta}}^{\prime}\right) \leq J_{i}(\underline{\hat{\theta}}) \quad \forall i \in\{1, \cdots, n\}$ and $\exists j \in\{1, \cdots, n\}$ such that $J_{j}\left(\underline{\hat{\theta}}^{\prime}\right)<J_{j}(\underline{\hat{\theta}})$. As a result of this and the fact that $w_{i} \geq 0$, it is readily derived using the form of $J(\underline{\theta} ; \underline{w})$ in (5) that $J\left(\underline{\hat{\theta}}^{\prime} ; \underline{w}\right)<J(\underline{\hat{\theta}} ; \underline{w})$. The last inequality implies that $\underline{\hat{\theta}}^{\prime}$, instead of $\underline{\hat{\theta}}$, is the global solution optimizing $J(\underline{\theta} ; \underline{w})$, which is a contradiction.

Thus, solving a series of single objective optimization problems of the type (5) and varying the values of the weights $w_{i}$ from 0 to 1 , excluding the case for which the values of all weights are simultaneously equal to zero, Pareto optimal solutions are alternatively obtained. These solutions for given $\underline{w}$ are denoted by $\underline{\hat{\theta}}(\underline{w})$. It should be noted, however, that there may exist Pareto optimal solutions that do not correspond to solutions of the single-objective weighted modal residuals problem. The existence of such Pareto solutions will be demonstrated in the application section 5 . Thus, the multi-objective identification method is essential if the whole Pareto front is to be obtained.

The single objective identification is computationally attractive since conventional minimization algorithms can be applied to solve the problem. However, a severe drawback of generating Pareto optimal solutions by solving the series of weighted singleobjective optimization problems by uniformly varying the values of the weights is that this procedure often results in cluster of points in parts of the Pareto front that fail to 
provide an adequate representation of the entire Pareto shape. Thus, alternative algorithms dealing directly with the multi-objective optimization problem and generating uniformly spread points along the entire Pareto front are preferred. Computational algorithms and related issues for solving the single-objective and the multi-objective optimization problems are discussed in Section 4.

\section{Model updating based on optimally weighted modal residuals}

The Pareto optimal models $\underline{\hat{\theta}}(\underline{w})$ along the Pareto front trade-off the fit between measured and model predicted modal data for different modal groups. The objective of this section is to address the problem of rationally selecting the optimal value $\underline{\hat{w}}$ of the weighting parameter set $\underline{w}=\left(w_{1}, \cdots, w_{n}\right)$ in (5) and subsequently estimating the most preferred structural model $\underline{\hat{\theta}}_{\text {opt }} \equiv \underline{\hat{\theta}}(\underline{\hat{w}})$ among the Pareto optimal models $\underline{\hat{\theta}}(\underline{w})$, utilizing the measured data and the selected model class. The decision for selecting a single most

preferred model $\hat{\theta}_{o p t} \equiv \underline{\hat{\theta}}(\underline{\hat{w}})$, among the Pareto optimal models $\underline{\hat{\theta}}(\underline{w})$, for further use in model-based prediction studies and reliability assessment, depends on the user preferences.

From the computational point of view, it is desirable that the selection of the most preferred optimal model does not necessitate the computation of the whole Pareto front and Pareto solutions, since this can be a very time consuming task for more than a few objectives and, therefore, such lengthy computations should be avoided. Moreover, it is desirable that the most preferred optimal model is not biased from measured modal properties that contain significant measurement and processing error or measured modal properties that cannot be well represented by the selected model class. For this, it is reasonable to weight the contribution of the modal group residuals $J_{i}(\underline{\theta})$ in the total 
residual measure (5) according to their residual error corresponding to the most preferred model. Specifically, modal groups with larger residual errors should be given less weight than modal groups with smaller residual errors. A rational choice is to select the weights to be inversely proportional to the values of the modal group residuals obtained for the most preferred model. Specifically, the $i$-th optimal weight value $\hat{w}_{i}$ could be chosen to be inversely proportional to the average value of the total residual error of the modal properties involved in the $i$-th modal group. That is, the optimal values $\hat{w}_{i}$ of the weights are chosen to satisfy the set of equations

$$
w_{i}=\alpha_{i} / J_{i}(\underline{\hat{\theta}}(\underline{w})), \quad i=1, \cdots, n
$$

where

$$
\underline{\hat{\theta}}(\underline{w})=\underset{\underline{\theta}}{\arg \min } J(\underline{\theta} ; \underline{w})
$$

is the optimal model parameter value that corresponds to the weight values $\underline{w}$, and $\alpha_{i}$ is the number of modal properties in the group $g_{i}$. In particular, for the two objective functions in (2), $\alpha_{1}=m$ and $\alpha_{2}=m N_{0}$. Using (6), the optimal weights in (5) are given by $\hat{w}_{i}=\alpha_{i} / J_{i}\left(\hat{\theta}_{o p t}\right)$, while the optimal value $\hat{\theta}_{o p t} \equiv \underline{\hat{\theta}}(\underline{\hat{w}})$ minimizes the optimally weighted residuals $J(\underline{\theta} ; \underline{\hat{w}})$ in (5), that is, $\underline{\hat{\theta}}_{\text {opt }} \equiv \underline{\hat{\theta}}(\underline{\hat{w}})$ is given by (7) for $\underline{w}=\underline{\hat{w}}$.

Since the most preferred Pareto optimal structural model is not known prior to the selection of the weights, the corresponding optimal values of the modal residual errors and so the optimal values of the weights are not known. Thus, the selection of the optimal weights should be made simultaneously with the selection of the optimal model so that at the optimum, the weight values are inversely proportional to the optimal residual errors as suggested in (6). Specifically, the optimal values $\underline{\hat{w}}$ and the most preferred Pareto 
optimal model $\underline{\hat{\theta}}_{\text {opt }} \equiv \underline{\hat{\theta}}(\underline{\hat{w}})$ are obtained by simultaneously solving the set of equations (6) and the optimization problem (7) with respect to $\underline{w}$ and $\underline{\theta}$. This is a nested optimization problem that is solved iteratively. Specifically, for each iteration on $\underline{w}$, required in satisfying (6), an optimization problem for estimating $\underline{\hat{\theta}}(\underline{w})$ needs to be solved.

It can be shown that the aforementioned problem is equivalent to the problem of finding $\hat{\theta}_{o p t}$ that minimizes the objective function

$$
I(\underline{\theta})=\sum_{i=1}^{n} \alpha_{i} \ln J_{i}(\underline{\theta})
$$

with respect to the parameter set $\underline{\theta}$ and then computing the optimal $\underline{\hat{w}}$ from $\hat{w}_{i}=\alpha_{i} / J_{i}\left(\hat{\theta}_{\text {opt }}\right)$. This can be readily verified by noting that the stationarity conditions $\left.\underline{\nabla}_{\underline{\theta}} I(\underline{\theta})\right|_{\underline{\theta}=\theta_{o p t}}=\underline{0}$ for the objective function $I(\underline{\theta})$ in (8), where $\underline{\nabla}_{\underline{\theta}}$ is the gradient vector with respect to $\underline{\theta}$, are given by

$$
\left.\frac{\partial I(\underline{\theta})}{\partial \theta_{j}}\right|_{\underline{\theta}=\underline{\theta}_{o p t}}=\left.\sum_{i=1}^{n} \frac{\alpha_{i}}{J_{i}\left(\underline{\theta}_{o p t}\right)} \frac{\partial J_{i}(\underline{\theta})}{\partial \theta_{j}}\right|_{\underline{\theta}=\underline{\theta}_{o p t}}=0, \quad j=1, \cdots, n
$$

which are exactly the same as the stationarity conditions $\left.\underline{\nabla} J(\underline{\theta} ; \underline{w})\right|_{\underline{\theta}=\underline{\hat{\theta}}(\underline{w})}=0$ for $(5)$ with $\underline{w}$ replaced by the optimal $\hat{w}_{i}=\alpha_{i} / J_{i}\left(\hat{\theta}_{o p t}\right)$ computed by (6). It should be noted that the direct optimization of $I(\underline{\theta})$ with respect to $\underline{\theta}$ is computationally much more efficient than the equivalent problem of solving simultaneously the set of equations (6) and the optimization problem (7).

The aforementioned method for selecting the most preferred model among the Pareto optimal models that satisfy (8) is referred to as the optimally weighted residual method. 
This choice corresponds to one out of the infinitely many Pareto optimal models. It is worth pointing out that the logarithmic estimator in (8) has also been shown to arise from a Bayesian statistical identification point of view [25]. Specifically, the most preferred optimal model is the most probable model that results asymptotically for large number of data from a Bayesian approach for structural identification. This Bayesian estimate is based on the assumption of Gaussian and independent errors between the measured modal properties and the corresponding modal properties predicted by the model class.

\section{Computational issues related to model updating formulations}

The proposed single and multi-objective identification problems are solved using available single and multi objective optimization algorithms. These algorithms are briefly reviewed and various implementation issues are addressed, including estimation of global optima from multiple local/global ones, as well as convergence issues.

\subsection{Single-objective identification}

The optimization of $J(\underline{\theta} ; \underline{w})$ in (5) with respect to $\underline{\theta}$ for given $\underline{w}$ and the optimization of $I(\underline{\theta})$ in (8) with respect to $\underline{\theta}$ can readily be carried out numerically using any available algorithm for optimizing a nonlinear function of several variables. These single objective optimization problems may involve multiple local/global optima. Conventional gradient-based local optimization algorithms lack reliability in dealing with the estimation of multiple local/global optima observed in structural identification problems $[25,30]$, since convergence to the global optimum is not guaranteed. Evolution strategies [31] are more appropriate and effective to use in such cases. Evolution strategies are random search algorithms that explore the parameter space for detecting the

neighborhood of the global optimum, avoiding premature convergence to a local 
optimum. A disadvantage of evolution strategies is their slow convergence at the neighborhood of an optimum since they do not exploit the gradient information. A hybrid optimization algorithm should be used that exploits the advantages of evolution strategies and gradient-based methods. Specifically, an evolution strategy is used to explore the parameter space and detect the neighborhood of the global optimum. Then the method switches to a gradient-based algorithm starting with the best estimate obtained from the evolution strategy and using gradient information to accelerate convergence to the global optimum.

Another issue that should be pointed out is that in order to guarantee the convergence of the gradient-based optimization methods for structural models involving a relatively large number of DOFs with several contributing modes, the gradient of the objective function with respect to the parameter set $\underline{\theta}$ has to be estimated accurately. It has been observed that numerical algorithms such as finite difference methods for gradient evaluation does not guarantee convergence due to the fact that the errors in the numerical estimation may provide the wrong directions in the search space and convergence to the local/global minimum is not achieved, especially for intermediate parameter values in the vicinity of a local/global optimum. Thus, the gradients of the objective functions should be provided analytically. For this, the gradients of the contributing modal frequencies and modeshapes, required in the estimation of the gradient of $J(\underline{\theta} ; \underline{w})$ in (5) or $I(\underline{\theta})$ in (8), are computed by expressing them exactly in terms of the values of the contributing modal frequencies, modeshapes and the gradients of the structural mass and stiffness matrices with respect to $\underline{\theta}$ using Nelson's method [32]. An advantage of the Nelson method is that the gradient of the modal frequencies and the modeshape vector of a specific mode can be computed from only the value of the modal frequency and the modeshape vector of 
the same mode, independently of the values of the modal frequencies and modeshape vectors of the rest of the modes.

\subsection{Multi-objective identification}

The set of Pareto optimal solutions can be obtained using available multi-objective optimization algorithms. Among them, evolutionary algorithms, such as the strength Pareto evolutionary algorithm [33], are well-suited to solve the multi-objective identification problem [24]. These algorithms process a set of promising solutions simultaneously and therefore are capable of capturing several points along the Pareto front. They are based on an arbitrary initialized population of search points in the parameter space, which by means of selection, mutation and recombination evolves towards better and better regions in the search space. The strength Pareto evolutionary algorithm does not require gradient information and it has the disadvantage of slow convergence, especially for objective vectors close to the Pareto front [24]. Moreover, it does not generate an evenly spread Pareto front, especially for large differences in objective functions.

A computationally more efficient algorithm for solving the multi-objective optimization problem is the Normal-Boundary Intersection (NBI) method [34]. The NBI algorithm produces an even spread of points along the Pareto front, even for problems for which the relative scaling of the objectives are vastly different. The NBI algorithm involves estimation of (a) the $n$ edge points of the Pareto front by solving $n$ unconstrained nonlinear optimization problems each one associated with the objective functions $J_{i}(\underline{\theta}), i=1, \cdots, n$, and (b) the points along the Pareto front by solving $N_{P}-n$ constrained nonlinear optimization problems, where $N_{P}$ is the total number of Pareto optimal solutions that are used to represent the Pareto front. The constrains involve the 
objective functions $J_{i}(\underline{\theta}), i=1, \cdots, n$. The $N_{P}-n$ constrained nonlinear optimization problems are solved using available gradient-based constrained optimization methods. However, it has been observed that in order to guarantee convergence, the gradients have to be estimated sufficiently accurately. Similarly to the single-objective identification case, finite difference methods for gradient evaluation of the constraints do not guarantee convergence. Herein, the gradients of the objective functions are provided analytically by computing analytically the gradients of the contributing modal frequencies and modeshapes using the Nelson's method [32].

Finally, it is of interest to compare the computational time involved for estimating the Pareto optimal solutions with the computational time required in conventional weighted residuals methods for estimating a single solution. This computational time is of the order of the number $N_{P}$ of points used to represent the Pareto front multiplied by the computational time required to solve a single-objective unconstrained or constrained optimization problem for computing each point on the front. However, for the NBI method, convergence can be greatly accelerated by using a good starting value for the constrained optimization problem close to the optimal value. This is achieved by selecting the Pareto optimal solution obtained from the current constrained optimization problem to be used as starting value for solving the constrained optimization problem for the next neighborhood point of the Pareto front. More details about the NBI method, advantages and drawbacks, can be found in reference [34].

\section{Application on a scaled three-story building structure}

Experimental data from a scaled three-story steel building structure are used to demonstrate the applicability and effectiveness of the proposed model updating methods, assess the effect of model error uncertainties on the variability of the Pareto optimal 
models, as well as investigate the response prediction accuracy and variability of the updated models.

\subsection{Description of the laboratory structure}

A schematic diagram of the side and the front views of the laboratory structure are given in Figure 1a. The floors of the building are made of identical steel beams of hollow orthogonal cross section. The two interstory columns that support each floor are made up of identical thin steel plates. The columns and beams are connected through angles with the help of bolts and nuts. The horizontal members are made to be much stiffer compared to the vertical structural elements so that the structural behaviour can be adequately represented by a shear beam building model. The total height of the structure is approximately $2.4 \mathrm{~m}$. The $y$ direction of the frame is made to be stiffer to prevent coupling of motion with the $x$ direction, the latter being the principal direction of interest. Detailed description and plans of the steel beams and columns can be found in Christodoulou [35]. The structure is considered as the reference structure and it is denoted by $\mathrm{C}_{0}$.

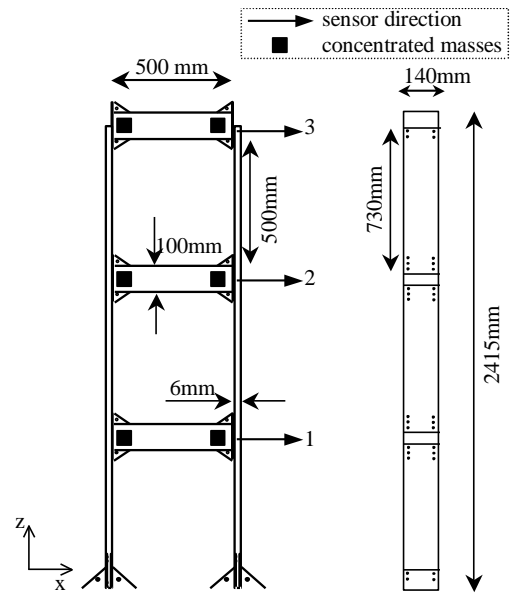

(a)

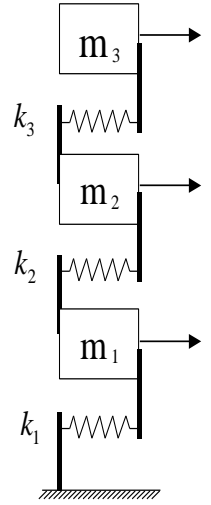

(b)

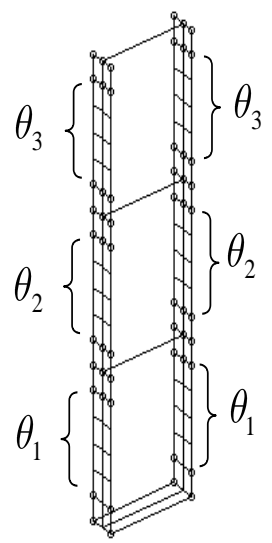

(c) 
Fig. 1. (a) Front and side views of 3-story building structure with added concentrated masses, (b)

parameterized 3-DOF model class (c) parameterized 546 DOF finite element model class.

A second structural configuration is considered by adding concentrated masses made from lead in both sides of each floor of the reference structure as shown in Figure 1a. The added weight due to the concentrated masses is approximately $9.5 \mathrm{Kg}$ per floor, while the total added mass corresponds to approximately $42 \%$ of the mass of the reference structure. The modified structural configuration with the concentrated masses is denoted by $C_{1}$.

\subsection{Modal identification}

The modal properties of the two structural configurations $C_{0}$ and $C_{1}$ are identified from frequency response functions that are obtained by processing the excitation force and acceleration response time histories generated from impulse hammer tests [36]. An array of three acceleration sensors located on the structure as schematically shown in Figure 1a, record the acceleration time histories during the test along the $x$ direction. Multiple data sets are generated and processed that correspond to different excitation position of the impulse hammer at the second and third floor of the structure along the $x$ direction. The common denominator least-squares complex frequency-domain method [37] is used to obtain the optimal values of the modal parameters assuming classically damped modes. Table 1 reports the values of the identified modal frequencies and modeshape components at the measured locations of the lowest three bending modes for the reference $C_{0}$ and mass modified $C_{1}$ structural configurations.

Table 1

Lowest three bending modal frequencies and modeshapes identified for the reference and the mass modified structural configurations.

\begin{tabular}{|l|l|}
\hline Reference Structure $C_{0}$ & Modified Structure $C_{1}$ \\
\hline
\end{tabular}




\begin{tabular}{|l|c|ccc|ccc|}
\hline \multicolumn{2}{|l|}{ Mode \# } & $1^{\text {st }}$ & $2^{\text {nd }}$ & $3^{\text {rd }}$ & $1^{\text {st }}$ & $2^{\text {nd }}$ & $3^{\text {rd }}$ \\
\hline \multicolumn{2}{|l|}{ Modal Freq. (Hz) } & 4.646 & 13.81 & 19.48 & 3.908 & 11.57 & 16.31 \\
\hline Modeshape & $1^{\text {st }}$ floor & 0.4561 & 1.000 & -0.7801 & 0.4408 & 1.000 & -0.7892 \\
\cline { 2 - 7 } Components & $2^{\text {nd }}$ floor & 0.8069 & 0.3009 & 1.000 & 0.8219 & 0.3528 & 1.000 \\
\cline { 2 - 6 } & $3^{\text {rd }}$ floor & 1.000 & -0.9026 & -0.6448 & 1.000 & -0.8709 & -0.5708 \\
\hline
\end{tabular}

\subsection{Parameterized model classes}

In order to investigate the effect of modelling error in model updating and model response prediction variability for each structural configuration, the following two parameterised model classes are introduced to represent the behaviour of the structure along the $x$ direction.

The first model class, which is schematically shown in Figure $1 \mathrm{~b}$, is a simple 3-DOF mass-spring chain model. The modelling is based on the assumptions that the floors of the structure are rigid and that the stiffness is provided by the interstory plates. A lumped mass model is considered. Specifically, the $i$-th mass of the model includes the mass of the $i$-th floor and half of the mass of the interstory plates that are attached to the $i$-th floor. Thus, based on the weights of the structural elements, the masses $m_{1}, m_{2}$ and $m_{3}$ are taken to be equal to $m_{1}=m_{2}=m_{0}$ and $m_{3}=0.76 m_{0}$, where $m_{0}=22.6 \mathrm{Kg}$. The initial (nominal) values of the spring stiffnesses $k_{01}, k_{02}$ and $k_{03}$ are taken to be equal, that is, $k_{01}=k_{02}=k_{03}=k_{0}$. The ratio $k_{0} / m_{0}$ was selected so as to minimize the difference between the first modal frequency predicted by the model and the first measured modal frequency for the structural configuration $C_{0}$.

The 3-DOF mass-spring chain model is parameterized introducing three parameters $\theta_{1}, \theta_{2}$ and $\theta_{3}$, one for the stiffness of each spring modelling the interstory stiffness, so that $k_{i}=\theta_{i} k_{0 i}$, for $i=1,2,3$, where $k_{0 i}=k_{0}$ is the nominal value of the stiffness of each spring in the nominal model and $k_{i}$ is the updated value of the stiffness of each 
parameterised spring based on the measured data. This parameterized model class is denoted by $M_{0}$. For the modified structure $C_{1}$ with added concentrated masses, the 3DOF model class is used with modified masses $m_{1}+m_{1}^{\prime}, m_{2}+m_{2}^{\prime}$ and $m_{3}+m_{3}^{\prime}$ that take into account the additional concentrated lead masses $m_{1}^{\prime}, m_{2}^{\prime}$ and $m_{3}^{\prime}$ added on the structure at each floor (see Figure 1a). The parameter set $\underline{\theta}$ is kept the same as the one used for the reference structure $C_{0}$. This parameterized model class for the modified structural configuration $C_{1}$ is denoted by $M_{1}$.

The second model class, which is schematically shown in Figure 1c, is a detailed finite element (FE) model. Each floor beam is modeled with a beam element, while the columns between each floor are modeled, due to its small thickness, with 12 plate elements each. The sizes of both types of elements are calculated from the structural drawing. The modulus of elasticity and the density are based on the material properties. The plate elements near the joints, between columns and floors, are assumed to be very stiff compared to the interstory plate elements, in order to model the large rigidity in these parts of the structure. The FE model developed based on modeling assumptions, the structural drawings and the properties of the materials used, is referred to as the initial (nominal) FE model. The total number of DOF is 546.

The 546-DOF finite element model is parameterised introducing three parameters $\theta_{1}$, $\theta_{2}$ and $\theta_{3}$, each one associated with the modulus of elasticity of the thin plate elements of first, second and third interstory columns, respectively, so that $E_{i}=\theta_{i} E_{0 i}$, for $i=1,2,3$, where $E_{0 i}=E_{0}$ is the nominal value of the modulus of elasticity of interstory plate elements in the initial FE model and $E_{i}$ is the updated value of the modulus of elasticity of each parameterised plate element. This parameterized model class is denoted by $M_{0, \mathrm{FE}}$. The FE model of the modified structure $C_{1}$ with the additional concentrated masses is 
obtained by modifying the FE model of the reference structure, adding in the FE model the known values of the concentrated lead masses at the edge nodes of the horizontal beam elements used to model the stiffness of the floors. The parameter set $\underline{\theta}$ is kept the same as the one used for the reference structure. This parameterized model class for the modified structural configuration $C_{1}$ is denoted by $M_{1, \text { EE }}$.

The model within each of the defined model classes with parameter values $\theta_{1}=\theta_{2}=\theta_{3}=1$ correspond to the initial (nominal) model of the model class. It should be emphasized that the three parameters $\theta_{1}, \theta_{2}$ and $\theta_{3}$ correspond to interstory stiffness properties of the three-story structure which are common for all four model classes introduced for the reference and mass modified configurations.

\subsection{Structural model updating}

Model updating results are computed for the model classes $M_{0}$ and $M_{0, \mathrm{FE}}$ based on the experimental data in Table 1 available for the reference structural configuration $C_{0}$. Similarly, model updating results are computed for the model classes $M_{1}$ and $M_{1, \mathrm{FE}}$ based on the experimental data in Table 1 available for the structural configuration $C_{1}$. The two objective functions in (2) are used for model updating. Thus, the objective space is two dimensional, while the parameter space is three dimensional. The Pareto optimal models are estimated from the proposed multiobjective identification method using the NBI algorithm and $N_{P}=20$ points along the Pareto front. The estimation of the optimal models corresponding to the optimally-weighted and equally weighted residuals methods, as well as the optimal models corresponding to the edge points of the Pareto front, is based on the hybrid optimization method combining evolution strategies and gradient based methods. 

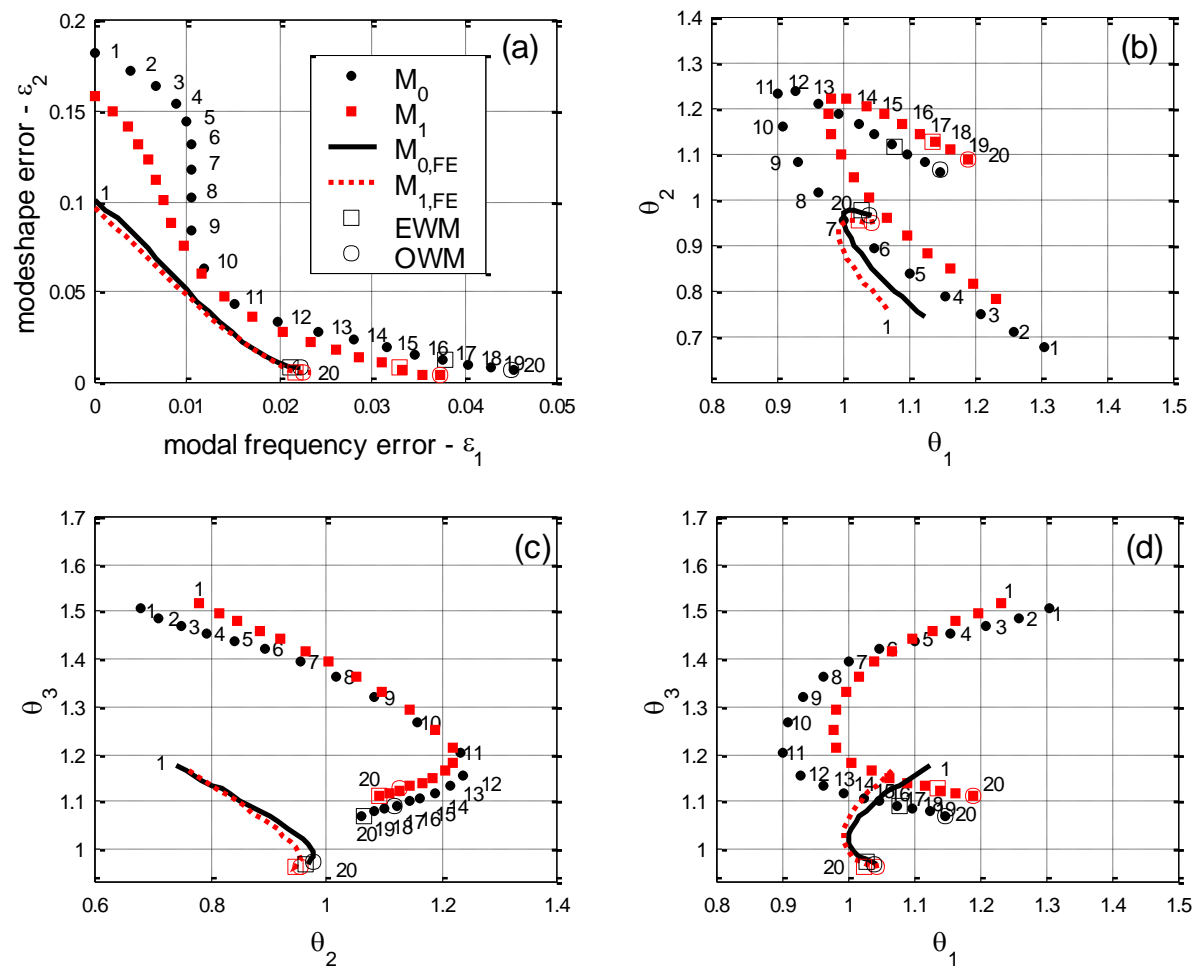

Fig. 2. Pareto front and Pareto optimal solutions in the (a) objective space and (b-d) parameter space, along with optimal solutions obtained from the optimally and equally weighted modal residuals methods.

The results from the multi-objective identification methodology are shown in Figure 2. For each model class and associated structural configuration, the Pareto front, giving the Pareto solutions in the two-dimensional objective space, is shown in Figure 2a. Specifically, the results in Figure $2 \mathrm{a}$ are given for the functions $\varepsilon_{1}(\underline{\hat{\theta}})=\sqrt{J_{1}(\underline{\hat{\theta}}) / \alpha_{1}}$ and $\varepsilon_{2}(\underline{\hat{\theta}})=\sqrt{J_{2}(\underline{\hat{\theta}}) / \alpha_{2}}$ which, given the definition of the objective functions in (2), represent a measure of the average errors of the modal properties involved in the two modal groups. The non-zero size of the Pareto front and the non-zero distance of the Pareto front from the origin are due to uncertainties arising from modeling and measurement errors. Specifically, the distance of the Pareto points along the Pareto front from the origin is an indication of the size of the overall measurement and modeling 
error. The size of the Pareto front depends on the size of the model error and the sensitivity of the modal properties to the parameter values $\underline{\theta}$ [25]. It is observed that the average errors $\varepsilon_{1}(\underline{\hat{\theta}})$ and $\varepsilon_{2}(\underline{\hat{\theta}})$ between the measured and the model predicted modal properties obtained from the Pareto optimal models $\underline{\hat{\theta}}$ for the higher fidelity 546-DOF model classes $M_{0, \mathrm{FE}}$ and $M_{1, \mathrm{FE}}$ are significantly smaller than the residual errors corresponding to the 3-DOF model classes $M_{0}$ and $M_{1}$. Consequently, for the higher fidelity 546-DOF model classes, the Pareto front moves closer to the origin of the objective space. In addition it is observed that the sizes of the Pareto fronts for the 546DOF model classes $M_{0, F E}$ and $M_{1, F E}$ reduce to approximately one third to half the sizes of the Pareto fronts observed for the 3-DOF model classes $M_{0}$ and $M_{1}$. These results certify, as it should be expected based on the modeling assumptions in Section 5.3, that the 546-DOF model classes are higher fidelity model classes than the 3-DOF model classes. Also the results in Figure 2a quantify the quality of fit, acceptance and degree of accuracy of a model class in relation to another model class based on the measure data.

Figures 2b-d show the corresponding Pareto optimal solutions in the threedimensional parameter space. For each model class, the Pareto optimal solutions are concentrated along a one-dimensional manifold in the three-dimensional parameter space. The Figures $2 b$-d show the projection of the Pareto solutions in the two-dimensional parameter spaces $\left(\theta_{1}, \theta_{2}\right),\left(\theta_{2}, \theta_{3}\right)$ and $\left(\theta_{1}, \theta_{3}\right)$. It is observed that a wide variety of Pareto optimal solutions are obtained for both model classes and structural configurations that are consistent with the measured data and the objective functions used. Comparing the Pareto optimal solutions for a model class, it can be said that there is no Pareto solution that improves the fit in both modal groups simultaneously. Thus, all Pareto solutions correspond to acceptable compromise structural models trading-off the fit in the modal 
frequencies involved in the first modal group with the fit in the modeshape components involved in the second modal group.

Comparing the Pareto front and Pareto optimal models for the 546-DOF model classes with the corresponding ones obtained for the 3-DOF model classes, it can be noted that the results are qualitatively similar. However, the size of the one dimensional optimal solutions manifolds for the 546-DOF model classes $M_{0, \mathrm{FE}}$ and $M_{1, \mathrm{FE}}$ are significantly smaller than the size of the manifolds for the 3-DOF model classes $M_{0}$ and $M_{1}$. These results clearly demonstrate that as the fidelity of the model class improves, the variability of the Pareto optimal models reduces. This has important implications in the selection of the weight values used in weighted modal residuals method for model updating and model-based prediction studies. Since the variability of the Pareto optimal solutions reduces as the fidelity of the models improves, the effect of the choice of weight on weighted modal residuals methods diminishes as the fidelity of the model increases.

Consider next the common parameter model classes $M_{0}$ and $M_{1}$ introduced for modeling the reference and modified structural configurations $C_{0}$ and $C_{1}$, respectively. The Pareto optimal values of the common parameter set $\underline{\theta}$ of the 3-DOF model classes $M_{0}$ and $M_{1}$ differ, despite the fact that the parameters for the two model classes $M_{0}$ and $M_{1}$ refer to the same interstory stiffnesses of the two different structural configurations. The differences can be attributed mainly to the size of modeling errors involved in the 3DOF model classes. Instead, comparing the Pareto optimal values obtained from the common parameter 546-DOF model classes $M_{0, \mathrm{FE}}$ and $M_{1, \mathrm{FE}}$ for the two structural configurations $C_{0}$ and $C_{1}$, it is observed that the optimal solution manifolds for the 546DOF model classes are significantly closer than the optimal solution manifolds for the 3DOF model classes. This certifies that the higher fidelity model classes provide 
consistent estimates of the common parameters in model classes introduced to model different structural configurations. Finally, it should be noted from the results in Figures 2b-d that the Pareto optimal values of the parameters predicted by the higher fidelity model classes $M_{0, \mathrm{FE}}$ and $M_{1, \mathrm{FE}}$ are significant different from the Pareto optimal values predicted by the simpler model classes $M_{0}$ and $M_{1}$. Thus, the model updating results and parameter estimates depend highly on the fidelity of the model class considered.

The optimal structural models corresponding to the optimally weighted (OWM) and the equally weighted (EWM) residuals methods for the 3-DOF and 546-DOF model classes are also shown in the Figure 2. It can be seen that these optimal models are points along the Pareto front, as it should be expected. The two methods, the OWM and the EWM, in general promote different Pareto optimal models for use in model-based prediction studies. For the example case considered, the Pareto solutions $\underline{\hat{\theta}}^{(\text {owm })}$ provided by the optimally weighted residuals method for the 3-DOF model classes are close to the boundary solution $\underline{\hat{\theta}}^{(20)}$ for both structural configurations $C_{0}$ and $C_{1}$. Also, the Pareto points corresponding to the optimally weighted (OWM) and the equally weighted (EWM) residuals methods for the 546-DOF model classes are also shown in the Figure 2 to be closer than the corresponding Pareto points for the 3-DOF model classes.
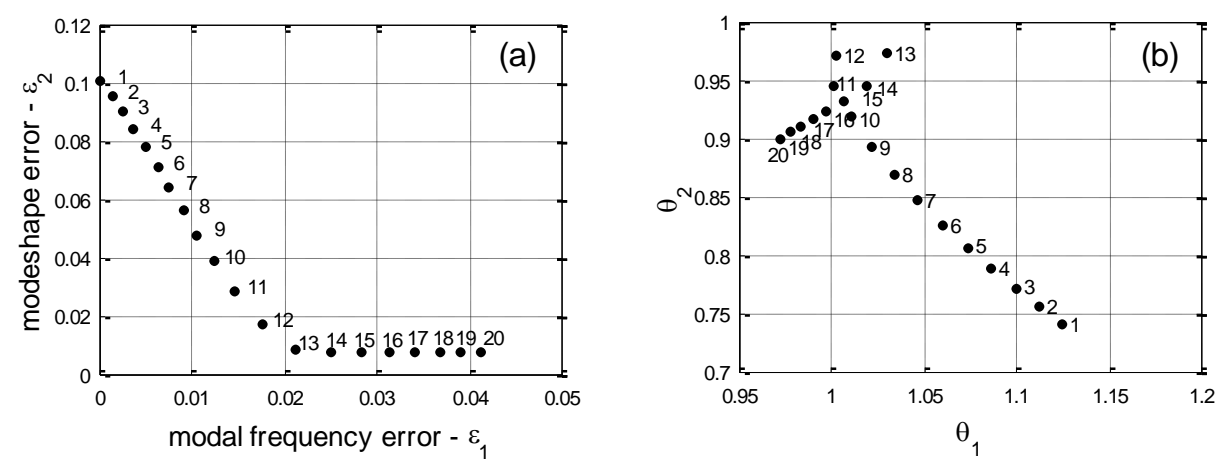

Fig. 3. (a) Pareto front, (b) projection of Pareto optimal solutions in $\left(\theta_{1}, \theta_{2}\right)$ plane for model class $M_{0, F E}$. 
Unidentifiability issues are next discussed which were ignored in presenting the Pareto front for the model class $M_{0, F E}$ in Figure 2a. Figure 3a shows the Pareto front obtained by the application of the proposed NBI algorithm for the 546-DOF model class $M_{0, F E}$. It is observed that there is an almost flat part of the Pareto front at the lower right edge of Figure 3a. This is due to the unidentifiability problems [28-29] encountered in estimating the optimal model corresponding to the right edge point of the Pareto front. In this case, the right edge point of the Pareto front is obtained by optimizing the function $J_{2}(\underline{\theta})$. It turns out that there is a lower dimensional manifold in the three-dimensional parameter space, shown in the two-dimensional projection $\left(\theta_{1}, \theta_{2}\right)$ in Figure $3 \mathrm{~b}$ to extend from point 13 to point 20, that give almost the same optimum for $J_{2}(\underline{\theta})$. Depending on the starting values of the parameter set $\underline{\theta}$, the gradient-based optimization algorithm converges to one of the infinite number of optimal models in this sub-manifold. As it is noted in Figure 3a, the flat unidentifiable portion of the Pareto front and the associated manifold in Figure $3 b$ are readily obtained by the NBI method. From the engineering point of view, the most important point from this flat portion is the most left point 13 in Figure 3a since all other points in the flat portion deteriorate the fit in the objective function $J_{1}(\underline{\theta})$ without significantly altering the fit in $J_{2}(\underline{\theta})$. In order to generate points only on the identifiable portion of the Pareto front for pre-selected number of points on it (e.g. 20 points as shown in Figure 2a), the analyst can repeat the application of the NBI algorithm with edge points of the Pareto front selected to be the points 1 and 13 in Figure 3. It should be noted that in the results presented in Figure 2 for the model classes $M_{0, \mathrm{FE}}$, only the identifiable part of the Pareto front is shown. 
It is worth mentioning that unidentifiable portions of the Pareto front were not observed for the other three model classes which implies that the problem was identifiable for all three model classes.

More careful examination of the optimal points resulted by the NBI method for the model class $M_{0}$ in Figure 2 reveals that the NBI points 6 and 7 for model class $M_{0}$ do not constitute Pareto points since it can be easily checked numerically that they do not satisfy conditions (4). Figure 4 shows the exact Pareto front and Pareto optimal solutions projected in the two-dimensional parameter space $\left(\theta_{1}, \theta_{2}\right)$ using NBI method for $N_{P}=40$ points. The NBI points that do not satisfy conditions (4) have been excluded from the Figures. It is clearly seen that the Pareto front in Figure 4a and the projection of the onedimensional Pareto solution manifold in $\left(\theta_{1}, \theta_{2}\right)$ plane in Figure $4 \mathrm{~b}$ is disconnected. The missing portion in Figure 4 that does not belong to Pareto front is the portion of the Pareto front and Pareto solutions in Figure 2 that extends approximately from point 5 to point 8. From the previous results, it is evident that the NBI method, despite the disconnected manifolds that exists, is capable of fully describing the Pareto solutions.
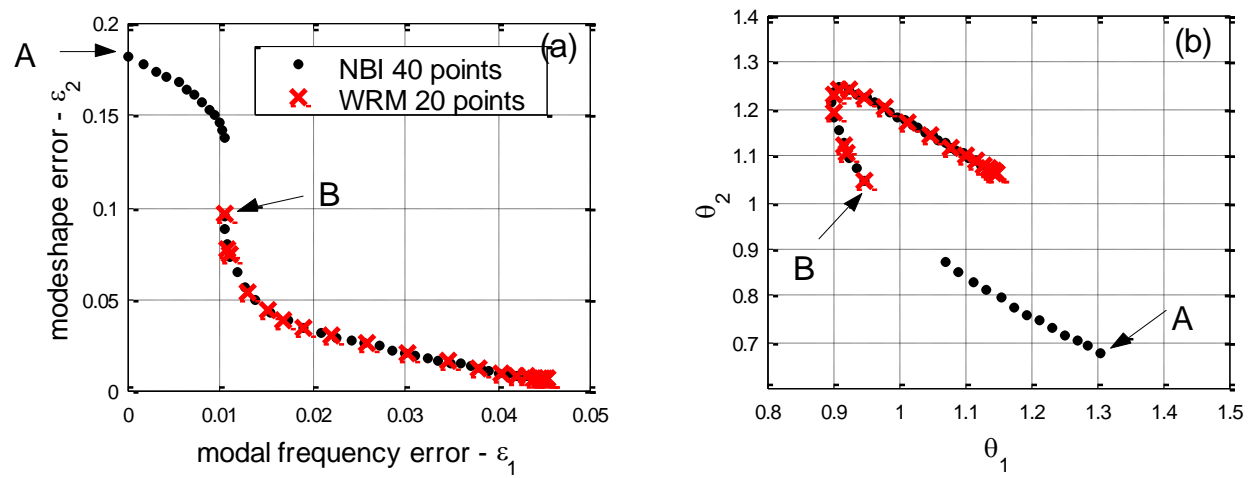

Fig. 4. (a) Pareto front, (b) projection of Pareto optimal solutions in $\left(\theta_{1}, \theta_{2}\right)$ plane for model class $M_{0}$. 
For comparison purposes, the weighted residuals method was also used to obtain the Pareto front by uniformly varying the weights in (5) from 0 to 1 . Specifically, dividing the interval $[0,1]$ for the weight $w_{1}$ into equally spaced sub-intervals using a step $\Delta w$, selecting the corresponding values of $w_{2}$ to satisfy the condition $w_{1}+w_{2}=1$, and estimating the optimal solutions for the $1 / \Delta w$ pairs of $\left(w_{1}, w_{2}\right)$ values, the Pareto front and the Pareto solutions are obtained and shown in Figures $4 \mathrm{a}$ and $4 \mathrm{~b}$ for model class $M_{0}$ for $\Delta w=0.05$. It can be seen that varying the weights in the weighted residuals method, part of the Pareto points can be completely missed. Specifically, the top left portion of the Pareto front in Figure 4a has been missed. Attempts to recover this part by fully exploring the region in the parameter space that corresponds to the top left part of the Pareto front have failed. Such attempts included the use of different values of the weights $\underline{w}$ in (5), different starting values for solving the single-objective optimization problems, various sizes of $\Delta w$, and an increasing number of Pareto points. Numerically there is no point in the top left part of the Pareto front that corresponds to a weight value in the weighted residual method. This is in accordance with the fact that there may be Pareto solutions that do not correspond to solutions of the single-objective optimization involving the weighted residuals norm (5) for any values of the weights. It should however be noted that for weight values $w_{1}=1$ and $w_{2}=0$, there is a global optimal solution that corresponds to the edge Pareto point A shown in Figure 4, as well as a local solution that corresponds to the edge Pareto point $\mathrm{B}$ of the left portion of the Pareto front. Both solutions, although one global and one local for the same weight values $w_{1}=1$ and $w_{2}=0$, are Pareto optimal solutions because they correspond to different values of the second objective $J_{2}(\underline{\theta})$. 
A final issue that is worth mentioning is that varying uniformly the weight values and computing the Pareto points using the weighted residuals method does not produce uniformly distributed points along the Pareto front. Instead, it may yield a cluster of points as it can be seen in Figure 4. As a consequence, part of the Pareto fronts can be misrepresented or completely missed.

\subsection{Prediction variability using Pareto optimal structural models}

The purpose of the identification is to construct faithful structural models, within a selected model class, that can be used for making improved structural performance predictions consistent with the measured data. The alternative models obtained along the Pareto front provide different performance predictions that are all acceptable based on the measured data and the measures of fit employed. The variability of these predictions is next explored.

The variability in the modal properties predicted by the Pareto optimal models is estimated for the model classes $M_{0}$ and $M_{0, F E}$ representing structural configuration $C_{0}$, and the model classes $M_{1}$ and $M_{1, F E}$ representing structural configuration $C_{1}$. The values of the three modal frequencies predicted by the Pareto optimal models from each model class, including the Pareto optimal models corresponding to the optimally weighted and equally weighted residuals methods, are shown in Figure 5. The measured modal frequencies for structural configurations $C_{0}$ and $C_{1}$ are also shown for comparison purposes. The variability of the corresponding MAC (Modal Assurance Criterion) values between the modeshape components predicted by the Pareto optimal models for each model class and the measured modeshapes for the three bending modes are shown in Figure 6. 

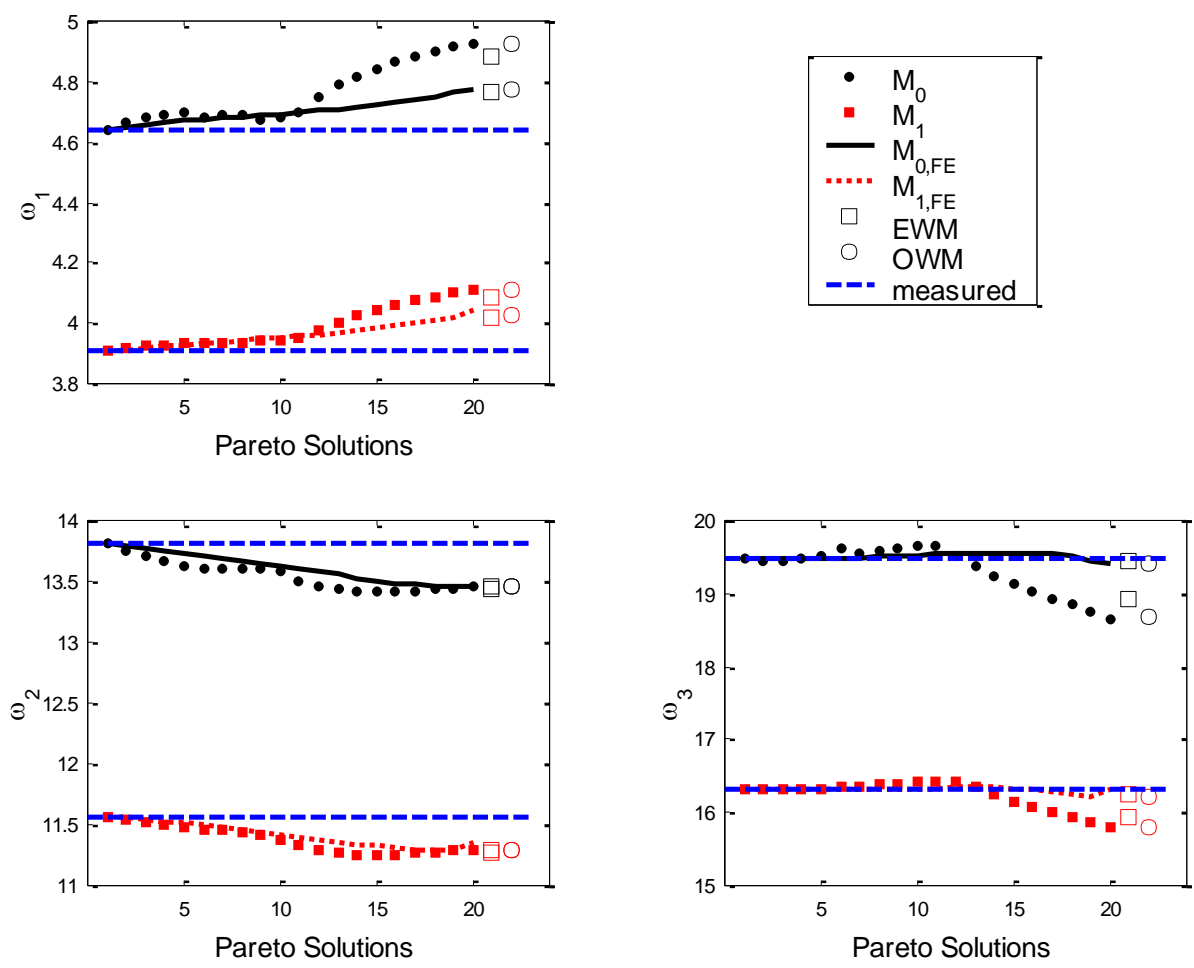

Fig. 5. Variability of modal frequencies predicted by the Pareto optimal solutions corresponding to model classes $M_{0}, M_{1}, M_{0, F E}$ and $M_{1, F E}$. The corresponding measured modal frequencies are also presented.

For each model class, different Pareto optimal models along the Pareto front result in different predictions of the structural modal frequencies and MAC values. A relatively large variability in the predictions is observed for the 3-DOF model classes $M_{0}$ and $M_{1}$. The maximum percentage error values between the Pareto optimal model predictions for the first, second and third modal frequency are respectively of the order of $6.0 \%, 2.7 \%$ and $4.9 \%$ for the model class $M_{0}$ and of the order of $5.1 \%, 2.3 \%$ and $3.1 \%$ for the model class $M_{1}$. The MAC values corresponding to the first, second and third mode vary respectively from $0.990,0.863$ and 0.851 for the model classes $M_{0}$ and from 0.997 , 0.895 and 0.884 for the model classes $M_{1}$ to values very close to 1.0 . 

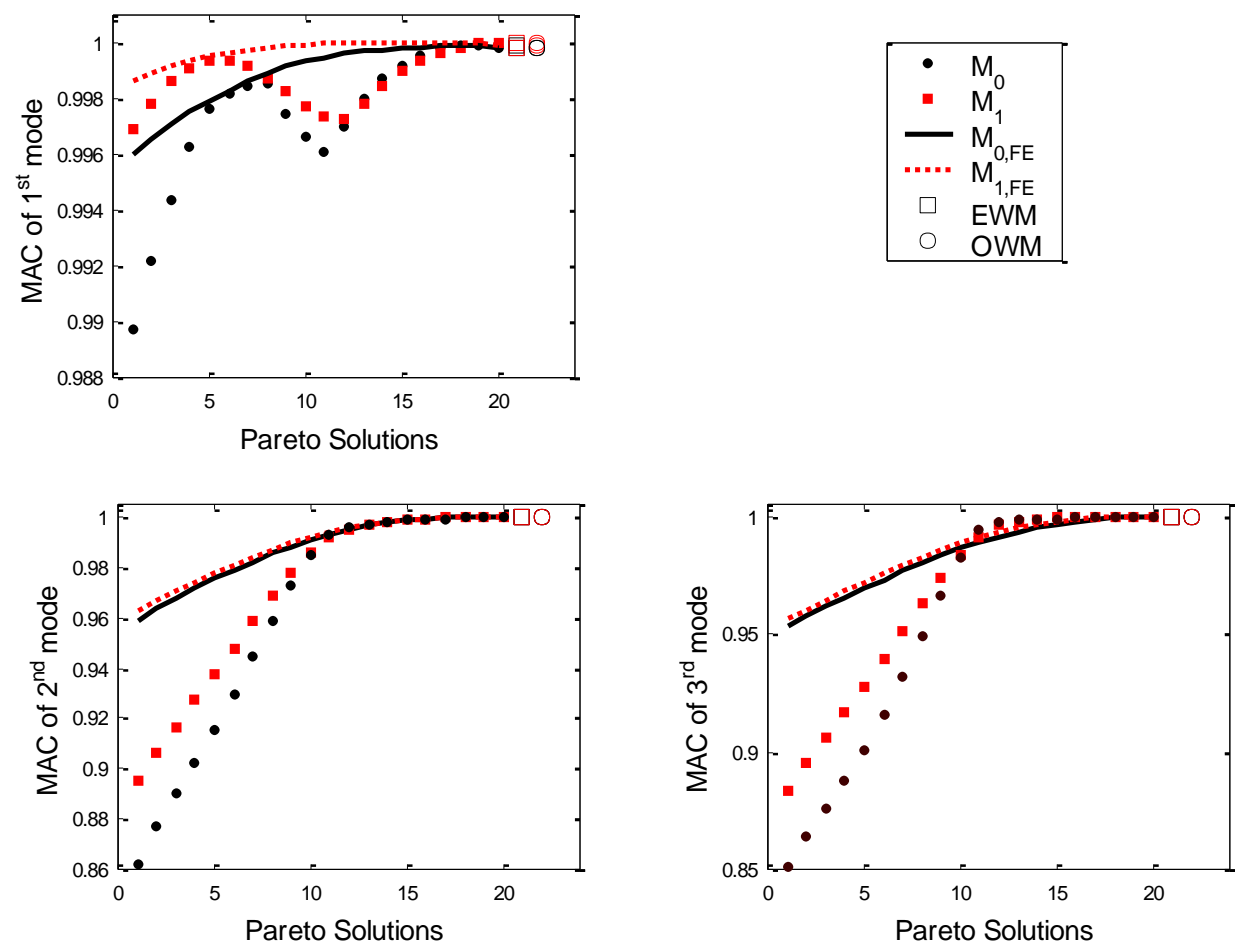

Fig. 6. Variability of MAC values predicted by the Pareto optimal solutions corresponding to model classes

$$
M_{0}, M_{1}, M_{0, F E} \text { and } M_{1, F E}
$$

It is clear that there is a trade off between the fit that the Pareto optimal models for model classes $M_{0}$ and $M_{0, \mathrm{FE}}$ provide to the modal frequencies and the modeshapes. Specifically, the Pareto models with small numbers close to Pareto point 1 provide a very good fit to the modal frequencies in the expense of deteriorating the fit in the MAC values to values significantly smaller than one. The Pareto models with large numbers close to Pareto point 20 for model classes $M_{0}$ and $M_{0, \mathrm{FE}}$ improve the MAC values to values very close to one in the expense of deteriorating the fit in the modal frequencies. Similar trade off in the fit is observed for the Pareto optimal models for model classes $M_{1}$ and $M_{1, F E}$. 
Comparing the predictions in Figure 5 from the 3-DOF model classes and the 546DOF model classes, the 546-DOF model classes $M_{0, \mathrm{FE}}$ and $M_{1, \mathrm{FE}}$ provide overall significantly better fit in the modal frequencies than the fit provided by the 3-DOF model classes $M_{0}$ and $M_{1}$. Also, comparing the results in Figure 6, it is observed that the higher fidelity 546-DOF model classes give MAC values between the Pareto optimal models and the measurements that are much closer to one than the MAC values obtained for the 3-DOF model class. These results verify that higher fidelity model classes tend to give better predictions that are less sensitive to selections required in model updating, such as the weight values used in weighted residuals methods.
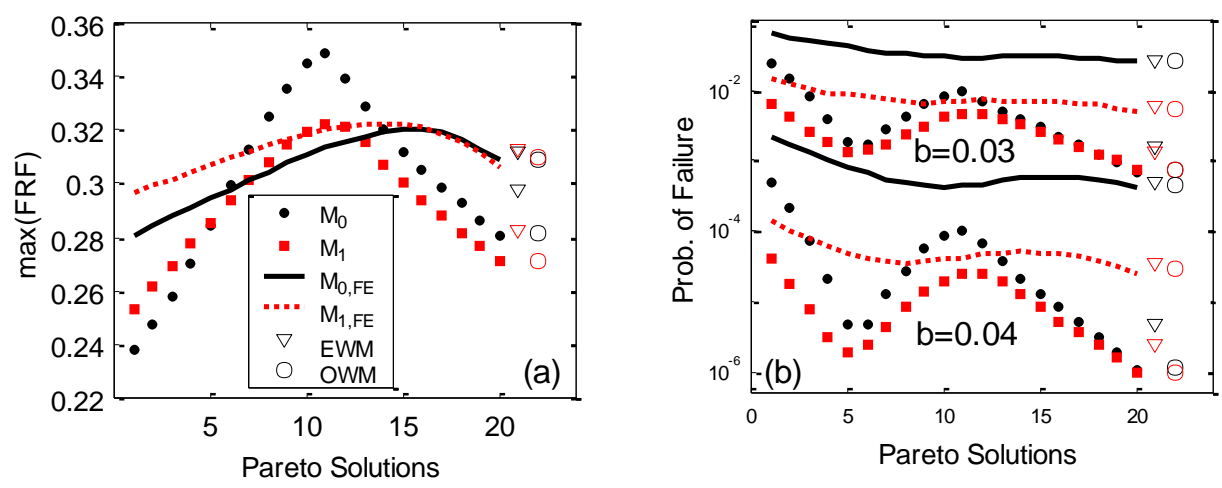

Fig. 7. (a) Variability of the maximum of the frequency response function at first floor, (b) the probability of failure corresponding to allowable interstory drift levels $b=0.03$ and $b=0.04$.

Similar variability can be constructed for other structural performance indices. Figure 7a shows the variability of the maximum of the frequency response function at the first floor obtained for the Pareto optimal models for all model classes for damping values of $\zeta=0.02$. Figure $7 \mathrm{~b}$ shows the variability of the probability of failure of the structure to uncertain stochastic loads. Herein, failure is defined as the condition for which either one of interstory floor drifts exceeds a level $b$. The failure probability estimates are obtained for white noise excitation of duration $T=30 \mathrm{sec}$, strength $S=0.001$ and for two 
allowable drift levels $b=0.03$ and $b=0.04$. The failure probability is obtained using efficient stochastic simulation methods available for linear systems [38-39] for 2000 samples. In particular, it is worth observing that a large variability in the maximum of the frequency response predictions, from [0.238 - 0.349] for the model class $M_{0}$ and [0.2810.321] for the model class $M_{0, F E}$, and in the failure probabilities from $\left[0.011 \times 10^{-4}-\right.$ $\left.5.1 \times 10^{-4}\right]$ for the model class $M_{0}$ and $\left[0.4 \times 10^{-3}-2.1 \times 10^{-3}\right]$ for the model class $M_{0, \mathrm{FE}}$, are observed for the Pareto optimal models. Similar variability levels are observed for model classes $M_{1}$ and $M_{1, F E}$. These variabilities are larger than the variabilities in the modal frequencies shown in Figures 5.

Comparing the results in Figure 7 for the 3-DOF and the 546-DOF model classes, it is observed that the variability in the predictions of the maximum of the frequency response function and the probability of failure due to allowable drift exceedance levels of the 546DOF model class is significantly smaller than the corresponding variability obtained from the 3-DOF model class.

Concluding, the predictions of the various performance indices from the Pareto optimal models may vary considerably. The variability in the predictions depends on the fidelity of the model class selected for identification. Higher fidelity model classes tend to reduce the variability in the predictions, diminishing the importance of selecting the weight values in weighted residuals methods.

\section{Conclusions}

Model updating algorithms were proposed to characterize and compute all Pareto optimal models from a model class, consistent with the measured data and the norms used to measure the fit between the measured and model predicted modal properties. The similarities with and differences from the conventional weighted modal residuals method 
were established. The most preferred Pareto optimal solution promoted by the proposed optimally weighted modal residuals method correspond to weight value for a modal group that is inversely proportional to the optimal residual between the measured and the model predicted properties involved in the modal group. The optimal values of the structural parameters for the most preferred Pareto optimal model are obtained by minimizing the sum of the logarithm of the modal residuals. The most preferred Pareto optimal model can be used for model-based predictions in case of more than a few objective functions for which the generation and visualization of Pareto optimal models in the multi-objective space is computationally less tractable. Hybrid algorithms based on evolution strategies and gradient methods are necessary and well-suited optimization tools for solving the resulting non-convex single-objective optimization problem and identifying the global optimum from multiple local ones. The proposed NBI method is well-suited for solving the multi-objective optimization problem and effectively computing the useful identifiable part of the Pareto front, as well as portion of the unidentifiable part of the Pareto front at the edge points.

Theoretical and computational issues were demonstrated by updating simple and higher fidelity model classes using experimental data from two configurations of a scaled three-story steel structure. A wide variety of Pareto optimal structural models consistent with the measured modal data was obtained. The variability in the Pareto optimal models is due to the model and measurement error. The large variability in the Pareto optimal models resulted in large variability in the response and structural reliability predictions. It has been demonstrated that higher fidelity model classes, tend to involve less model error, move the Pareto front towards the origin and reduce the size of the Pareto front in the objective space, reduce the variability of the Pareto optimal solutions, provide better fit to the measured quantities, and give much better predictions corresponding to reduced 
variability. In particular, as the fidelity of the model class improves, the importance of selecting the weight values in weighted residuals methods diminishes.

Future work will explore the effectiveness of the multi-objective identification method for damage detection purposes by monitoring changes in the Pareto optimal models using the measured modal properties from healthy and damage structural states.

\section{Acknowledgements}

This research was co-funded by the Greek Ministry of Education and the European Community within the Hrakleitos program framework under grant MIS: 88730.

\section{References}

[1] M. Baruch, I.Y. Bar Itzhack, Optimal weighted orthogonalization of measured modes, AIAA Journal 16 (4) (1978) 346-351.

[2] J.E. Mottershead, M.I. Friswell, Model updating in structural dynamics: A survey, Journal of Sound and Vibration 167 (1993) 347-375.

[3] C. Farhat, P.M. Hemez, Updating finite element dynamics models using an element-by-element sensitivity methodology, AIAA Journal 131 (1993) 1702-1711.

[4] D. Capecchi, F. Vestroni, Identification of finite-element models in structural dynamics, Engineering Structures 15 (1) (1993) 21-30.

[5] M. Link, Updating analytical models by using local and global parameters and relaxed optimization requirements, Mechanical Systems and Signal Processing 12 (1) (1998) 7-22.

[6] K.V. Yuen, J.L. Beck, L.S. Katafygiotis, Unified probabilistic approach for model updating and damage detection, Journal of Applied Mechanics - Transactions of the ASME 73 (4) (2006) 555-564. 
[7] S.W. Doebling, C.R. Farrar, M.B. Prime, A summary review of vibration-based damage identification methods, The Shock and Vibration Digest 30 (2) (1998) 91 105.

[8] I. Takewaki, M. Nakamura, Stiffness-damping simultaneous identification under limited observation, Journal of Engineering Mechanics (ASCE) 131 (10) (2005) $1027-1035$.

[9] J.L. Beck, L.S. Katafygiotis, Updating models and their uncertainties- I: Bayesian statistical framework, Journal of Engineering Mechanics (ASCE) 124 (1998) 455461.

[10] C. Papadimitriou, J.L. Beck, L.S. Katafygiotis, Updating robust reliability using structural test data, Probabilistic Engineering Mechanics 16 (2001) 103-113.

[11] J.L. Beck, S.K. Au, Bayesian updating of structural models and reliability using Markov chain Monte Carlo simulation, Journal of Engineering Mechanics (ASCE) 128 (2002) 380-391.

[12] H. Sohn, K.H. Law, Bayesian probabilistic approach for structural damage detection, Earthquake Engineering and Structural Dynamics 26 (1997) 1259-1281.

[13] C.P. Fritzen, D. Jennewein, T. Kiefer, Damage detection based on model updating methods, Mechanical Systems and Signal Processing 12 (1) (1998) 163-186.

[14] F. Vestroni, D. Capecchi, Damage detection in beam structures based on frequency measurements, Journal of Engineering Mechanics (ASCE) 126 (7) (2000) 761-768.

[15] M.W. Vanik, J.L. Beck, S.K. Au, Bayesian probabilistic approach to structural health monitoring, Journal of Engineering Mechanics (ASCE) 126 (2000) 738-745.

[16] M.I. Friswell, J.E. Mottershead, Inverse methods in structural health monitoring, Damage Assessment of Structures - Key Engineering Materials 204 (2) (2001) 201210. 
[17] D. Bernal, B. Gunes, Flexibility based approach for damage characterization: benchmark application, Journal of Engineering Mechanics (ASCE) 130 (1) (2004) $61-70$.

[18] H.F. Lam, L.S. Katafygiotis, N.C. Mickleborough, Application of a statistical model updating approach on phase I of the IASC-ASCE structural health monitoring benchmark study, Journal of Engineering Mechanics (ASCE) 130 (1) (2004) 34-48.

[19] A. Teughels, G. De Roeck, Damage detection and parameter identification by finite element model updating, Archives of Computational Methods in Engineering 12 (2) (2005) 123-164.

[20] K.V. Yuen, J.L. Beck, Reliability-based robust control for uncertain dynamical systems using feedback of incomplete noisy response measurements, Earthquake Engineering and Structural Dynamics 32 (5) (2003) 751-770.

[21] K.D. Hjelmstad, S. Shin, Crack identification in a cantilever beam from modal response, Journal of Sound and Vibration 198 (1996) 527-545.

[22] K.F. Alvin, Finite element model update via Bayesian estimation and minimization of dynamic residuals, AIAA Journal 35 (1997) 879-886.

[23] K. Bohle, C.P. Fritzen, Results obtained by minimizing natural frequencies and MAC-value errors of a plate model, Mechanical Systems and Signal Processing 17 (2003) 55-64.

[24] Y. Haralampidis, C. Papadimitriou, M. Pavlidou, Multi-objective framework for structural model identification, Earthquake Engineering and Structural Dynamics 34 (6) (2005) 665-685. 
[25] K. Christodoulou, C. Papadimitriou, Structural Identification Based on Optimally Weighted Modal Residuals, Mechanical Systems and Signal Processing 21 (2007) 4-23.

[26] L.S. Katafygiotis, Treatment of model uncertainties in structural dynamics, Technical Report EERL91-01, California Institute of Technology, Pasadena, CA., 1991.

[27] L.S. Katafygiotis, J.L. Beck, Updating models and their uncertainties. II: Model identifiability, Journal of Engineering Mechanics (ASCE) 124 (4) (1998) 463-467.

[28] L.S. Katafygiotis, C. Papadimitriou, H.F. Lam, A probabilistic approach to structural model updating, International Journal of Soil Dynamics and Earthquake Engineering 17 (1998) 495-507.

[29] L.S. Katafygiotis, H.F. Lam, Tangential-projection algorithm for manifold representation in unidentifiable model updating models, Earthquake Engineering and Structural Dynamics 31 (4) (2002) 791-812.

[30] A. Teughels, G. De Roeck, J.A.K. Suykens, Global optimization by coupled local minimizers and its application to FE model updating, Computers and Structures 81 (24-25) (2003) 2337-2351.

[31] H. G. Beyer, The theory of evolution strategies, Berlin, Springer-Verlag, 2001.

[32] R.B. Nelson, Simplified calculation of eigenvector derivatives, AIAA Journal 14 (9) (1976) 1201-1205.

[33] E. Zitzler, L. Thiele, Multi-objective evolutionary algorithms: A Comparative case study and the strength Pareto approach, IEEE Transactions on Evolutionary Computation 3 (1999) 257-271. 
[34] I. Das, J.E., Jr., Dennis, Normal-Boundary Intersection: A new method for generating the Pareto surface in nonlinear multi-criteria optimization problems, SIAM Journal of Optimization 8 (1998) 631-657.

[35] K. Christodoulou, Methodology for Structural Identification and Damage Detection, $\mathrm{PhD}$ Thesis Report SDL-06-01, Department of Mechanical and Industrial Engineering, University of Thessaly, 2006.

[36] D.J. Ewins, Modal Testing - Theory Practice and Applications, 2nd edn, Research Studies Press, UK, 2000.

[37] P. Verboven, Frequency-Domain System Identification for Modal Analysis, PhD Thesis, Vrije Universiteit Brussel, Belgium, 2002.

[38] S.K. Au, J.L. Beck, First excursion probability for linear systems by very efficient importance sampling, Probabilistic Engineering Mechanics 16 (3) (2001) 193-207.

[39] K.V. Yuen, L.S. Katafygiotis, An efficient simulation. method for reliability analysis of linear dynamical systems using simple additive rules of probability, Probabilistic Engineering Mechanics 20 (1) (2005) 109-114. 